\title{
ECOLOGIA DE MOSQUITOS (DIPTERA, CULICIDAE) EM CRIADOUROS NATURAIS E ARTIFICIAIS DE ÁREA RURAL DO NORTE DO ESTADO DO PARANÁ, BRASIL. VI. COLETAS DE LARVAS NO PERIDOMICÍLIO
}

\author{
José Lopes ${ }^{1}$
}

\begin{abstract}
ECOlogy OF MOSQUitofs (Diptera, Cui.ICIDAE) IN NATURAL AND ARTIficial rural breEding places in Northern Parana State, Brazil. VI. Larvae COLLECTIONS IN THEIR HOME SURROUNDINGS. In containers found in home surroundings Aedes fluviatilis, Anopheles albitarsis, Anopheles argyritarsis, Anopheles evansae, Anopheles strodei, Culex bahamensis, Culex bigoti, Culex coronator, Culex eduardoi, Culex mollis, Culex quinquefasciatus, Culex sp., Limatus durhamii and Psorophora cingulata larvae were collected. The most densely colonized containers were water tanks, cement trough and tyres. Among the most frequent species a meaningful population variation to Culex quinquefasciatus and Aedes fluviatilis, the first being more frequent during the spring and the second during the summer. Anopheles albitarsis, Anopheles evansae, Anopheles strodei, Culex bahamensis e Culex sp. were only collected in large- sizer cement containers. The other species did not show any particular preferences.

KEY WORDS. Culicidae, larvae, artificial places breeding
\end{abstract}

A destruição dos ecótopos naturais associada ao assentamento humano nestes locais, favoreceu a aproximação e o convívio do homem com os culicídeos, o que facilita o aparecimento de zoonoses.

O descarte pelo homem de recipientes com capacidade de reter água, favorece a procriação de grupos de indivíduos de uma população de mosquitos, dotados de plasticidade genética que permita o seu desenvolvimento nestes criadouros artificiais.

A presente pesquisa objetivou conhecer as espécies de Culicidae que procriam em recipientes encontrados no peridomicílio rural do Norte do Paraná; evidenciar os recipientes mais aceitos pelas diferentes espécies; contribuir com o conhecimento da ecologia desses culicídeos, verificando influências de fatores físicos ambientais e flutuação populacional.

\section{MATERIAL E MÉTODOS}

A figura 1 dá uma idéia do padrão geral dos peridomicílios que foram estudados nesta pesquisa. A área de estudo está localizada nos municípios de Cambé

1) Departamento de Biologia Animal e Vegetal, Universidade Estadual de Londrina. Caixa Postal 6001, 86051-970 Londrina, Paraná, Brasil. 
e Lóndrina, no Norte do Estado do Paraná. Mais especificamente, acompanha toda a extensão do Ribeirão São Domingos. Este ribeirão tem a sua nascente no município de Cambé e a foz no ribeirão Cafezal, já no município de Londrina. O seu leito apresenta uma extensão de aproximadamente $11 \mathrm{~km}$. As residências estavam alocadas a uma distância aproximada de $0,3 \mathrm{~km}$ daquele ribeirão.

Foram determinadas quatro sedes dos sítios como locais de estudo, distantes aproximadamente $2 \mathrm{~km}$ entre uma e outra. Este espassamento permitiu que os pontos de coleta ficassem distribuídos equidistantemente nos $11 \mathrm{~km}$ de extensão do ribeirão.

As coletas tiveram seu início no mês de junho de 1988 e sucederam-se até junho de 1989, obedecendo periodicidade mensal e todos os criadouros artificiais que continham água foram examinados.

As larvas de quarto instar e as pupas foram aprisionadas com penerinha de coar chá com malha de $1 \mathrm{~mm}^{2}$, daí retiradas com conta gotas e transferidas para frasco de transporte. A amostra máxima retirada de cada criadouro, foi constituída de $100 \mathrm{ml}$ de água do recipiente, contendo as larvas, acumulada pela somatória dos lances com o conta-gotas executados para a captura dos imaturos que estavam retidos na peneirinha. No laboratório as larvas e as pupas foram criadas para obtenção dos adultos. A identificação foi baseada nestes adultos e nas suas respectivas exúvias, eliminadas por ocasião da muda.

\section{RESULTADOS E DISCUSSÃO}

A tabela I relaciona as espécies de Culicidae coletadas nos diferentes tipos de recipientes encontrados com água no peridomicílio rural. Este resultado é um aglomerado de todos os pontos de coleta.

As caixas de água mostram o maior índice de positividade com $73,9 \%$ das observações sendo positivas, seguidas pelos pneus com $63,2 \%$ e cochos com $43,8 \%$ de positividade.

Para análise da entomofauna culicideana, optou-se somente pelos recipientes mais produtivos.

Foram coletadas quatorze espécies diferentes de Culicidae, onde Anopheles (Nyssorhynchus) argyritarsis Robineau-Devoidy, 1827, Culex (Culex) grupo coronator Dynar \& Knab, 1827, Culex (Culex) eduardoi Casal \& Garcaia, 1968, Culex (Culex) quinquefasciatus Say, 1823, Limatus durhamii Theobald, 1901 e Psorophora (Grabhamia) cingulata Fabricius, 1805, tiveram distribuição em todos pontos analisados. A ampla distribuição por toda área de coleta pode indicar tratar-se de espécies adaptadas ao ambiente antropogênico.

Anopheles (Nyssorhynchus) albitarsis Lynch Arribalzaga, 1878 e Culex sp. foram específicos de caixa de água. A este grupo pode se incluir Anopheles (Nyssorhyncus) evansae (Bréthes, 1925), Anopheles (Nyssorhyncus) strodei Root, 1926 e Culex (Culex) bahamensis Dyar \& Knab, 1906 que foram encontradas, em caixa d'água e também em cocho de cimento, ficando os dois grupos de espécies, com exceção de Anopheles evansae, restritos ao ponto 1. Os dois reservatórios de água apresentam a mesma constituição. As outras espécies apresentaram maior colonização nos diferentes tipos de criadouros. 


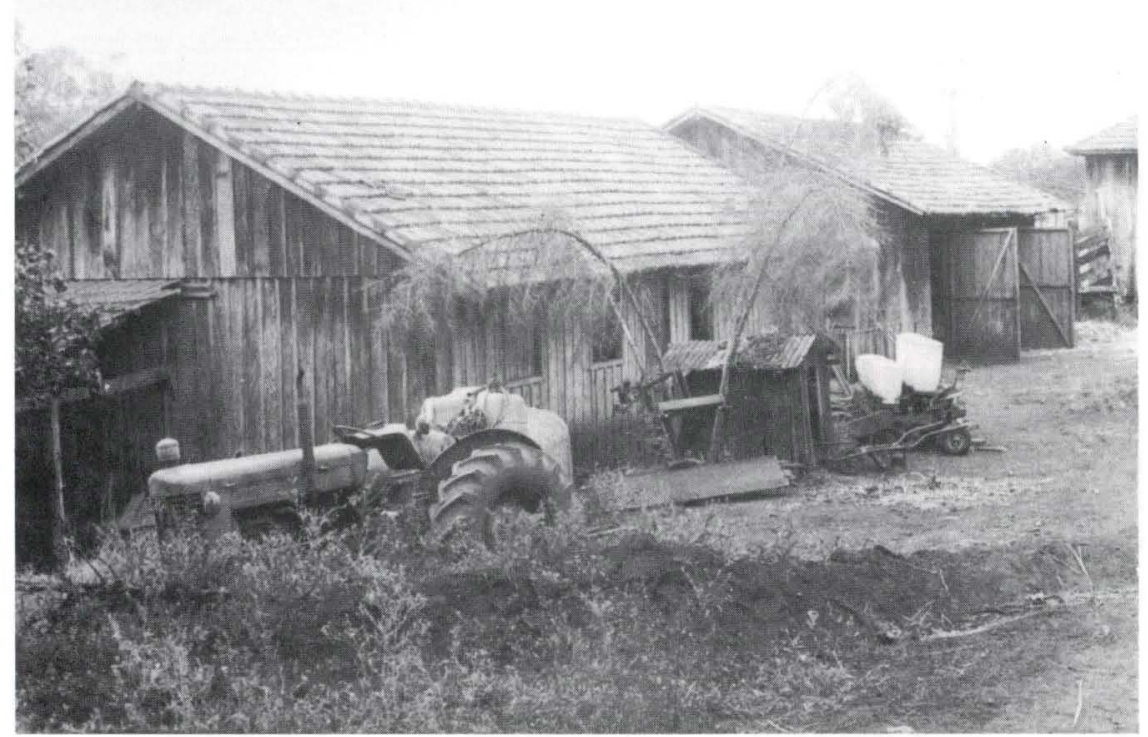

Fig. 1. Aspecto geral do peridomicilio no ponto de coleta número 1.

Tabela I. Espécies de Culicidae coletadas nos diferentes tipos de recipientes encontrados no peridomicílio rural dos Municípios de Cambé e Londrina (Paraná), no período compreendido entre VI/88 a VI/1989. (+) Recipiente positivo, (-) recipiente negativo.

\begin{tabular}{|c|c|c|c|c|c|c|c|c|c|c|c|c|}
\hline Espécies & $\begin{array}{r}\text { Caixa } \\
\text { d'água }\end{array}$ & Cocho & opneu & Vala & $\begin{array}{l}\text { a Vaso } \\
\text { sanitário }\end{array}$ & $\begin{array}{l}\text { Tambor } \\
\text { peite }\end{array}$ & $\begin{array}{c}\text { r Implemento } \\
\text { agricola }\end{array}$ & Lata & $\begin{array}{c}\text { Pia } \\
\text { esmaltada }\end{array}$ & Telha & Panela & $\begin{array}{l}\text { Recipiente } \\
\text { plástico }\end{array}$ \\
\hline Aedes fluviatilis & + & + & + & - & - & - & - & - & - & - & - & - \\
\hline Anopheles albitarsis & + & - & - & - & - & - & - & - & - & - & - & - \\
\hline Anopheles argyritarsis & + & + & + & + & - & - & - & - & - & - & - & - \\
\hline Anopheles evansae & + & + & - & - & - & - & - & - & - & - & - & - \\
\hline Anopheles strodei & + & + & - & - & - & - & - & - & - & - & - & - \\
\hline Culex bahamensis & + & + & - & - & - & - & - & - & - & - & - & - \\
\hline Culex bigoti & + & + & + & + & - & - & - & - & - & - & - & - \\
\hline Culex coronator & + & + & + & + & - & - & - & - & - & - & - & - \\
\hline Culex eduardoi & + & + & + & + & - & - & - & - & - & - & - & - \\
\hline Culex mollis & + & - & + & + & - & - & - & - & - & - & - & - \\
\hline Culex quinquefasciatus & + & + & + & + & - & + & - & - & - & - & - & - \\
\hline Culex sp. & + & - & - & - & - & - & - & - & - & - & - & - \\
\hline Limatus durhamii & - & + & + & - & + & - & - & - & - & - & - & - \\
\hline Psorophora cingulata & + & - & + & + & + & - & - & - & - & - & - & - \\
\hline
\end{tabular}

Foi aplicado o teste de Análise de Variância objetivando detectar a existência ou não de preferências pelas espécies por algum dos criadouros. Antes de aplicar o referido teste, o valor obtido em cada coleta, foi transformado em raíz $(x+0.5)$ para homogenizar a amostra. Culex quinquefasciatus e Anopheles argyritarsis não mostram significância. Aedes (Ochlerotatus) fluviatilis (Lutz, 1904) e Culex grupo coronator tiveram, como resultado, uma variação significativa a nível de $5 \%$. A primeira espécie mostrou preferência por cocho de cimento e a segunda por caixa de água. 
Culex quinquefasciatus foi a espécie mais freqüente em pneu e também apresentou a maior densidade populacional. O mesmo ocorre em caixa de água.

Em cocho de cimento Aedes fluviatilis é o mais abundante, deixando Culex quinquefasciatus com a segunda posição. Em termos de freqüência, Culex coronator predomina neste tipo de ambiente.

Em vala com água, Culex coronator é o mais freqüente e abundante, vindo logo a seguir Culex (Culex) mollis Dyar \& Knab, 1906 em termos de freqüência e Psorophora cingulata, em abundância, com 39 exemplares em uma única coleta. Esta concentração faz com que esta seja a terceira pela Média de Willians, pois Culex mollis com 19 exemplares coletados em três oportunidades fica como a segunda mais abundante, por esta média.

Constatações de poucas espécies predominantes em buraco de árvore e em pneus foram feitas JENKINS \& CARPENTER (1946), MitchELl \& ROCKETT (1981) e BEIER et al. (1983).

Anopheles albitarsis foi coletado uma única vez em uma caixa d'água grande, onde também Culex bahamensis foi mais freqüente. A primeira espécie reproduz-se preferencialmente em lagoas com vegetação e com incidência solar direta (LANE 1936; CERQUEIRA 1961). Esta única ocorrência em caixa d'água pode ter sido meramente ocasional. A segunda espécie é relatada como encontrada criando-se em criadouros artificiais.

Anopheles argyritarsis foi mais freqüente em cocho de cimento. Primitivamente, este anofelineo cria-se em lagoas com as mesmas características das descritas para Anopheles albitarsis (CERQUEIRA 1961), todavia esta espécie é considerada euriécia, pois é freqüentemente encontrada em diferentes tipos de recipientes.

Para Culex grupo coronator, cabe ressaltar sua presença em $100 \%$ das coletas na poça de água e sua alta freqüência em cocho, principalmente em um que sempre se encontrava com pouca água, cheio de matéria orgânica em decomposição e à sombra. Resultados semelhantes foram observados por LOURENÇO-DE-OLIVEIRA et al. (1986). A colonização de diversos tipos de recipientes foi observada por LOPES et al. (1993). Em condições naturais larvas deste mosquito foram coletadas em brejo, nascentes, lagoas e riachos e folhas caídas (LANE 1936; CERQUEIRA 1961; LOzOVEI \& LuZ 1976). Observando a fisionomia dos criadouros natural e comparando-se aos artificiais preferídos pela espécie, pode-se pensar em um direcionamento evolutivo de aceitação de diferentes tipos de criadouro, partindo de água no solo com vegetação para criadouros artificiais que continham no seu interior uma grossa camada de matéria orgânica e pouca água, à semelhança de condições naturais. Nas caixas com volume maior de água e menos matéria orgânica, a densidade de larvas foi baixa, indicando condições ainda pouco aceitas.

Culex mollis e Psorophora cingulata mostram maior freqüência em poça de água, o que poderia evidenciar condições mais primitivas. Estas duas espécies, no entanto, são freqüentemente citadas como colonizando recipientes artificiais (FoRATTINI et al. 1987; LOPES et al. 1993).

Culex quinquefasciatus está amplamente distribuído, mas com baixa porcentagem de freqüência apenas em poça. Esta espécie é amplamente conhecida como 
sinantrópica.

Culex (Lutzia) bigoti Bellardi, 1862, teve freqüência semelhante em caixa de cimento e poça. Observação desta espécie em pneu foi relatada por LoPEs et al. (1993) e em poças de água com ou sem vegetação por LANE (1936).

Limatus durhamii teve freqüência relativamente baixa e não foi constatada em poça de água. Este sabetíneo coloniza recipientes que estejam na sombra, associados com alguma vegetação (LOPES et al. 1985, 1987, 1993).

A tabela II relaciona o total de larvas para cada espécie, a média de Willians e a porcentagem de abundância para as diferentes espécies nos diferentes tipos de recipientes.

Tabela II. Número de larvas de Culicidae nos diferentes tipos de criadouros, Média de Willians $(X w)$ e a porcentagem de abundância larváriapara cada espécie, no peridomicílio da área rural nos Municipios de Cambé e Londrina (Paraná), de VI/88 a VI/1989.

\begin{tabular}{|c|c|c|c|c|c|c|c|c|c|c|c|c|}
\hline \multirow{2}{*}{ Espécies } & \multicolumn{3}{|c|}{ Pneu } & \multicolumn{3}{|c|}{ Cocho } & \multicolumn{3}{|c|}{ Caixa d'água } & \multicolumn{3}{|c|}{ Vala } \\
\hline & Total & $X_{w}$ & $\%$ & Total & $X w$ & $\%$ & Total & $X_{w}$ & $\%$ & Total & $X w$ & $\%$ \\
\hline Aedes fluviatilis & 1076 & 0,84 & 26,20 & 1280 & 1,29 & 46,89 & 175 & 0,61 & 5,20 & 0 & 0,00 & 0,00 \\
\hline Anopheles albitarsis & 0 & 0,00 & 0,00 & 0 & 0,00 & 0,00 & 1 & 0,02 & 0,03 & 0 & 0,00 & 0,00 \\
\hline Anopheles argyritarsis & 5 & 0,07 & 0,12 & 63 & 0.43 & 2,30 & 225 & 0,48 & 6.60 & 4 & 0,19 & 1,36 \\
\hline Anopheles evansae & 0 & 0.00 & 0.00 & 5 & 0,06 & 0,18 & 7 & 0,09 & 0,21 & 0 & 0,00 & 0,00 \\
\hline Anopheles strodei & 0 & 0,00 & 0,00 & 5 & 0,05 & 0,18 & 2 & 0,03 & 0,06 & 0 & 0,00 & 0,00 \\
\hline Culex bahamensis & 0 & 0,00 & 0,00 & 1 & 0,02 & 0,04 & 45 & 0,26 & 1,32 & 0 & 0,00 & 0,00 \\
\hline Culex bigoti & 22 & 0,15 & 0,54 & 16 & 0,13 & 0,59 & 39 & 0,30 & 1,20 & 1 & 0,06 & 0,34 \\
\hline Culex coronator & 8 & 0,09 & 0,19 & 212 & 0,77 & 7,77 & 382 & 0,77 & 11,20 & 228 & 1,49 & 77,60 \\
\hline Culex eduardoi & 20 & 0,17 & 0,49 & 28 & 0,22 & 1,03 & 6 & 0,08 & 0,18 & 1 & 0,06 & 0,34 \\
\hline Culex mollis & 18 & 0,13 & 0,44 & 0 & 0,00 & 0,00 & 6 & 0,06 & 0,20 & 19 & 0,49 & 6,46 \\
\hline Culex quinquefasciatus & 2896 & 1,38 & 70,40 & 1109 & 1,26 & 40,62 & 2508 & 1,14 & 73.80 & 2 & 0,10 & 0,68 \\
\hline Culex sp. & 0 & 0,00 & 0,00 & 0 & 0.00 & 0,00 & 1 & 0,02 & 0,03 & 0 & 0,00 & 0,00 \\
\hline Limatus durhamii & 15 & 0,13 & 0,36 & 5 & 0,07 & 0,18 & 1 & 0,02 & 0,03 & 0 & 0,00 & 0,00 \\
\hline Psorophora cingulata & 52 & 0,16 & 1,26 & 6 & 0,06 & 0,22 & 1 & 0,02 & 0,03 & 39 & 0,32 & 13,27 \\
\hline Total & 4112 & & 100,00 & 2730 & & 100,00 & 3399 & & 100,00 & 294 & & 100,00 \\
\hline Total de observaçס̄es & & 21 & & & 15 & & & 17 & & & 5 & \\
\hline
\end{tabular}

Pela tabela III, observa-se que Aedes fluviatilis apresentou maior índice larvário de janeiro a março, Anopheles argyritarsis em outubro, janeiro e fevereiro e Culex bigoti em setembro, Culex coronator em setembro, outubro e fevereiro. Culex quinquefasciatus tem distribuição durante todo o ano e sua maior densidade populacional de outubro a janeiro, com exceção do mês de agosto onde houve uma queda brusca na sua presença, com pico populacional em outubro.

Para constatação de flutuação populacional, o Teste de Análise de Variância foi aplicado nos dados mencionadas na tabela III, referentes às quatro espécies mais frequêntes. Os dados foram transformados em raiz $(x+0.5)$ para homogeneização da amostra e referem-se aos resultados obtidos no conjunto de todos os tipos de criadouros. Os resultados foram significativos ao nivel de $5 \%(\mathrm{P}<0,05)$ para Aedes fluviatilis e Culex quinquefasciatus e não significativo para Anopheles argyritarsis e Culex coronator.

No contraste das médias, pelo Teste de Duncan, obtidas na estatística anterior, obteve-se para Aedes fluviatilis que os meses mais produtivos são os de fevereiro e março e os meses menos produtivos são os de agosto e outubro. Esta 
maior abundância nos meses mais quentes e mais chuvosos do ano, coincide com os registros de Lozovei \& Luz (1976) e Silva \& Lopes (1985). Para Culex quinquefasciatus o Teste de Duncan seleciona os meses de outubro e novembro como os mais produtivos. A partir daí, há um decréscimo quase linear e voltando a crescer em julho do próximo ano. Essa espécie foi a mais frequente de junho a janeiro, sendo sucedida por Aedes fluviatilis de fevereiro a maio. Estes resultados sugerem que Culex quinquefasciatus seja dotado de maior tolerância ao abaixamento da temperatura, quando comparada com Aedes fluviatlis.

Tabela III. Total mensal e Média de Willians (XW) dos Culicidae com maior freqüência em criadouros no peridomicílio em área rural nos Municipios de Cambé e Londrina (Paraná), de $\mathrm{VI} / 88$ a VI/1989.

\begin{tabular}{lccccccccccccc}
\hline \multicolumn{1}{c}{ Espécies } & Jun & Jul & Ago & Set & Out & Nov & Dez & Jan & Fev & Mar & Abr & Mai & Jun \\
\hline Aedes fluviatilis & 345 & 435 & 0 & 1 & 0 & 135 & 32 & 291 & 561 & 504 & 109 & 12 & 106 \\
Xw & 0,88 & 0,83 & & 0,10 & & 0,36 & 0,60 & 1,13 & 1,63 & 1,44 & 0,97 & 0,56 & 0,84 \\
Anopheles argyritarsis & 1 & 0 & 6 & 2 & 19 & 14 & 19 & 30 & 187 & 14 & 5 & 0 & 0 \\
Xw & 0,06 & & 0,17 & 0,16 & 0,67 & 0,20 & 0,26 & 0,64 & 0,84 & 0,24 & 0,36 & & \\
Culex coronator & 46 & 90 & 120 & 27 & 94 & 68 & 71 & 64 & 118 & 66 & 66 & 0 & 0 \\
Xw & 0,33 & 0,53 & 0,42 & 0,86 & 1,47 & 0,58 & 0,56 & 0,70 & 0,85 & 0,56 & 0,46 & & \\
Culex quinquefasciatus & 529 & 706 & 548 & 233 & 1460 & 2256 & 231 & 378 & 124 & 38 & 0 & 3 & 9 \\
Xw & 1,56 & 1,77 & 0,54 & 1,37 & 2,39 & 1,88 & 1,11 & 1,70 & 0,72 & 0,50 & & 0,30 & 0,28 \\
\hline
\end{tabular}

Na Análise de Regressão Linear entre os fatores Média de Willians para cada espécie, das quatro mais freqüentes, com temperatura e precipitação pluviométrica, obteve-se significância a nível de 5\% $(\mathrm{P}<0,05)$ para Anopheles argyritarsis com a temperatura e a precipitação $(r=0,6203+0,2086 ; r=0,7301+0,1817$, respectivamente) e para Culex coronator com a temperatura $(\mathrm{r}=0,5988+0,2254)$. As duas espécies não mostraram flutuação populacional anual, mas são diretamente influenciadas por estes fatores físicos.

Para a distribuição das quatro espécies mais freqüentes, de acordo com as estações do ano (Tab. IV), foi aplicado o Teste de Análise de Variância, com os dados transformados em raiz ( $x+0,5)$, o qual mostrou-se significativo a nível de $5 \%$ $(\mathrm{P}<0,05)$ para Aedes fluviatilis e Culex quinquefasciatus. O Teste de Duncan separa, como significativamente mais produtivo, o verão e a primavera, respectivamente. Pelo Qui-quadrado, a primeira espécie não mostrou diferença entre as médias no outono e inverno, sendo significativamente inferior na primavera. Para Culex quinquefasciatus a média observada no inverno não difere da esperada e no verão e outono é significativamente inferior à média esperada.

Para Anopheles argyritarsis e Culex coronator a análise de variância foi não significativa, e o qui-quadrado mostra a primeira como outra espécie predominante no verão, estando próximo ao esperado na primavera e com populações muito baixas no outono e inverno. Culex coronator apresentou média significativamente superior à média esperada no inverno, não diferenciando na primavera e bem inferior no outono.

DAVIS (1945) afirmou que a flutuação populacional está na dependência de água no solo. Dependendo das chuvas, as populações flutuam de ano para ano, de estação para estação e de mês para mês. Nesta pesquisa, as flutuações devem estar 
mais relacionadas à temperatura ambiental que exerce influência sobre a fisiologia da reprodução, já que a maioria dos criadouros eram mantidos com água.

Destas espécies, a sucessão mais nítida observada é a de Aedes fluviatilis sobre Culex quinquefasciatus e Culex coronator. Culex quinquefasciatus é sucedido a partir do mês de janeiro e Culex coronator já é superado por Aedes fluviatilis a partir do mês de novembro. A temperatura ambiente pode ter influenciado o comportamento reprodutivo das fêmeas.

Tabela IV. Média de Willians para as espécies no peridomicilio da zona rural dos Município de Cambé e Londrina (Paraná) conforme as estações do ano (1988/1989).

\begin{tabular}{lcccc}
\hline \multicolumn{1}{c}{ Espécies } & Primavera & Verăo & Outono & Inverno \\
\hline Aedes fluviatilis & 0,32 & 1,40 & 0,79 & 0,31 \\
Anopheles argyritarsis & 0,38 & 0,57 & 0,12 & 0,17 \\
Anopheles evansae & 0,16 & 0,03 & 0,00 & 0,05 \\
Anopheles strodei & 0,10 & 0,00 & 0,00 & 0,00 \\
Culex bahamensis & 0,15 & 0,00 & 0,03 & 0,17 \\
Culex bigoti & 0,22 & 0,13 & 0,12 & 0,16 \\
Culex coronator & 0,87 & 0,70 & 0,15 & 0,60 \\
Culex eduardoi & 0,17 & 0,13 & 0,12 & 0,12 \\
Culex mollis & 0,07 & 0,10 & 0,04 & 0,00 \\
Culex quinquefasciatus & 1,79 & 0,79 & 0,19 & 1,22 \\
Limatus durhamii & 0,00 & 0,21 & 0,00 & 0,00 \\
Psorophora cingulata & 0,00 & 0,33 & 0,10 & 0,00 \\
\hline
\end{tabular}

AGRADECIMENTOS. Ao Dr. Oswaldo Paulo Forattini e a Dra. Maria Anice Mureb Sallum pela confirmação das identificações específicas dos Culicidae.

\section{REFERÊNCIAS BIBLIOGRÁFICAS}

Beier, J.C.; M. Travis; C. Patricoski \& J.K. Feder. 1983. Influence of water chemical and environmental parameters on larval mosquito dynamics in tires. Jour. Med. Entomol. 12 (1): 76-80.

CERQueIRA, N.L. 1961. Distribuição geográfica dos mosquitos da Amazônia. Revta bras. Ent. 10: 111-168.

DAVIS, D.E. 1945. The anual cycles of plants, mosquitoes, birds and mammals in two Brasilian forest. Ecol. Monogr. 15 (3): 243-295.

Forattini, O.P.; A. DE C. Gomes; D. Natal; I. Kakitani \& D. MuricCi. 1987. Freqüência domiciliar e endofilia de mosquitos Culicidae no Vale do Ribeira, São Paulo, Brasil. Rev. Saúde Pública 21 (3): 188-192.

Jenkins, D.W. \& S.J. CARPENTER. 1946. Ecology of the tree hole breeding mosquitoes of Neartic North America. Ecol. Monogr. 16: 33-47.

LANE, J. 1936. Notas sobre culicideos de Matto Grosso. Revta Mus. Paulista 20: 173-210.

Lopes, J.; J.R. Arias \& J.D. Charlwood. 1985. Estudo Ecológico de Culicidae (Diptera) silvestres criando em pequenos recipientes de água em mata e em capoeira no município de Manaus - Am. Ciênc. e Cult. 37 (8): 1299-1340.

Lopes, J.; A.M. Borsato \& M.A. Pires. 1987. Entomofauna da Mata Godoy. I. 
Culicidae (Diptera) procriando-se em criadouros artificiais introduzidos na mata. Semina 8 (2): 67-69.

Lopes, J.; M.A.N. DA Silva; A.M. Borsato; V.D.R. DE OliveIra \& F.J. DE A. OLIVEIRA. 1993. Aedes (Stegomyia) aegypti L. e a culicideofauna associada em área urbana da região sul, Brasil. Rev. Saúde Pública 27 (5): 326-333.

LOURENÇO-DE-OliveIRA, R.; R. HeYdEN \& T.F. DA Silva. 1986. Alguns aspectos da ecologia de mosquitos (Diptera: Culicidae) de uma área de planície (Granjas Calabria), em Jacarepaguá, Rio de Janeiro. V. Criadouros. Mem. Inst. Oswaldo Cruz 81 (3): 265-271.

LozoveI, A. \& E. Luz. 1976. Diptera Culicidae em Curitiba e arredores. I. Ocorrência. Arq. Biol. Tecnol., Curitiba, 19: 25-42.

MitCHELL, L. \& C.L. RocheTT. 1981. An investigation of the larval habitat of five specie of tree-hole breeding mosquitoes (Diptera: Culicidade). Great Lakes Entomol. 14: 123-129.

SiLVA, M.A.N. DA \& J. LoPES. 1985. Dados sobre a potencialidade criadoura de Culicidae (Diptera) do Cemitério Sao Pedro - Londrina - Paraná. Semina, Londrina, 6 (3): 133-139.

Recebido em 26.IX.1996; aceito em 03.IX.1997. 